\title{
Motor Using Mechanical Vibration of Multiple Bimorph Beams
}

\author{
Hiroyuki Yaguchi ${ }^{1}$ \\ ${ }^{1}$ Faculty of Engineering, Tohoku Gakuin University, Miyagi, Japan \\ Correspondence: Hiroyuki Yaguchi, Faculty of Engineering, Tohoku Gakuin University, Miyagi, Japan. Tel: \\ 81-22-368-7104. E-mail: yaguchi@mail.tohoku-gakuin.ac.jp
}

Received: May 17, 2017

Accepted: May 23, 2017

Online Published: May 31, 2017

doi:10.5539/mer.v7n1p7

URL: https://doi.org/10.5539/mer.v7n1p7

\begin{abstract}
The present paper proposes a non-magnetic motor with a rotor rotated by the mechanical resonance energy of four bimorph cantilever beams excited by an electrostatic force. The use of a flexible material such as silicon rubber enables conversion of translational vibration to rotary movement in one direction. The rotational speed of the proposed motor increases in proportion to the input voltage when two bimorph beams are used, and the maximum rotational speed was found to be $6,804 \mathrm{rpm}$ when the input voltage was set to $24.6 \mathrm{~V}$. Next, the basic characteristics of a prototype motor with four bimorph cantilever beams, including rotational speed, output torque, and efficiency, were determined experimentally. The experimental results revealed that a maximum rotational speed of $6,370 \mathrm{rpm}$ was obtained when the output torque was $19.6 \mu \mathrm{Nm}$. The proposed motor was also observed to produce an output torque of $63.7 \mu \mathrm{Nm}$ when the rotational speed was $1,491 \mathrm{rpm}$. The maximum efficiency was $6.2 \%$ when the input power was 0.3 $\mathrm{W}$. For the proposed motor, the volume and weight were reduced by approximately $35 \%$, as compared with a motor from a previous study.
\end{abstract}

Keywords: motor, frictional force, flexible material, bimorph cantilever beam, resonance, second vibration mode

\section{Introducation}

The electromagnetic motor is used widely in medical, welfare and industry. However, an output torque is quite small when the size of the motor becomes small. Therefore, a micro-gear is necessary to increase torque of the motor. In turn, this would require a reducer manufactured with very high precision. On the other hand, non-magnetic motors do not use electromagnets or permanent magnets, and so do not produce electromagnetic waves. More specifically, non-magnetic motors perform stably in high-magnetic-field environments. The demand has increased for non-magnetic motors for generating high torque in strong magnetic fields generated through superconductivity, as well as in medical applications such as magnetic resonance imaging (MRI) and magnetoencephalography. Non-magnetic motors have been discussed for long time. As such, many studies have investigated the mechanisms of motors using non-magnetic devices, such as electrostatic elements (Jian et al., 2006; Xiaolong et al., 2014; Peled et al., 2016; Aoyagi et al., 2016; Yu et al., 2016; Yamamoto et al., 2005; Kurosawa, 1997; Niino et al., 1997), electrorheological fluid (Yokota et al., 2004), shape memory alloys (Sharma et al., 2008), magnetostrictive elements (Pack et al., 2013; Ueno, 2009), the electrostatic optical motor by using PLZT element (Morikawa et al., 2003), and the combination of a frictional force and an electromagnetic force (Maruno et al., 2000; Yaguchi et al., 2014). An ultrasonic motor is being developed as a typical example of a non-magnetic motor, and the design and production techniques of this ultrasonic motor are almost established. However, ultrasonic motors are very expensive, although the cycle of use is short. By another means a stick-type non-magnetic motor manufactured using two bimorph cantilever beams has also been developed (Yaguchi, 2015). The rotation principles and properties of this motor have been demonstrated experimentally. However, this motor is not small.

In the present paper, a thin-type motor of smaller size that combines an electrostatic force of non-magnetic force and a mechanical resonance was proposed. Multiple bimorph cantilever beams were examined in trial manufacture. Multiple bimorph cantilever beams were constructed in order to achieve resonance at the second mode of vibration, and use of a flexible material, such as silicon rubber, enabled conversion of translational vibration to rotary movement in one direction. In an experiment, the rotational properties of two bimorph cantilever beams with silicon rubber at the tips were examined. A thin-type motor with two and four bimorph cantilever beams was fabricated, and the influence of each parameter on the output torque and efficiency was investigated. Consideration of newly connection method for multiple cantilever beams to increase generating force of the motor carried out in this paper. The volume and weight of the motor was reduced by approximately $35 \%$, as compared with the previous motor (Yaguchi, 2015). 


\section{Structure of Motor having two Bimorph Cantilever Beams}

Figure 1(a) and 1(b) show a schematic diagram of the proposed thin-type motor coupled by two bimorph beams. The motor consists of two bimorph cantilever beams with silicon rubber attached to their tips, a rotor, a bearing, and a steel ball. The size of bimorph beam used in this study was designed to be very small compared with previous study (Yaguchi, 2015). Case of one bimorph beam, no large force is generated. Therefore, two bimorph cantilever beams were connected at the antinode of the vibration node as shown in Figure 1 (c). Thus, consideration of newly connection method for multiple cantilever beams to increase generating force of the motor carried out in this paper. The beams are connected by a steel ball (diameter: $0.2 \mathrm{~mm}$ ), and exhibit mechanical resonance due to an electrostatic force. The resonance energy rotates the rotor using the frictional drive generated by the silicon rubber. The bimorph cantilever beam is composed of two piezoelectric ceramic test pieces of $2 \mathrm{~mm}$ in width, $16 \mathrm{~mm}$ in length (L), and $0.055 \mathrm{~mm}$ in thickness. A Ni alloy piece having a width of $2 \mathrm{~mm}$, a length of $20 \mathrm{~mm}$, and a thickness of $0.06 \mathrm{~mm}$, as shown in Figure 2. Silicon rubber $2 \mathrm{~mm}$ in length, $0.5 \mathrm{~mm}$ in thickness, and $1.5 \mathrm{~mm}$ in width was used in the present study, as shown in Figure 2. The proposed motor rotates the rotor by picking the silicon rubber, as shown in Figure 3. The rotational principle and simple theoretical formula of the proposed motor has been demonstrated in study (Yaguchi, 2015).

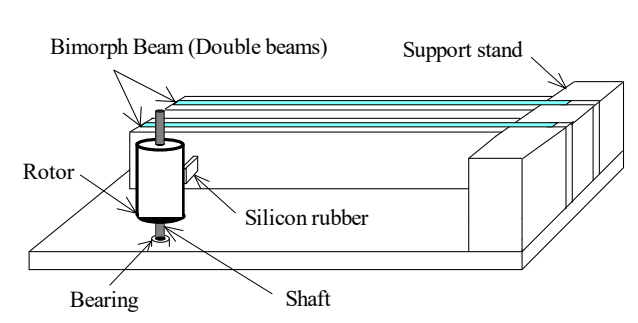

(a) Outline of motor using two bimorph beams

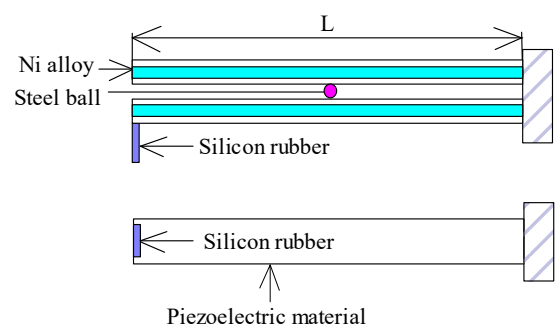

(b) Double cantilever beams

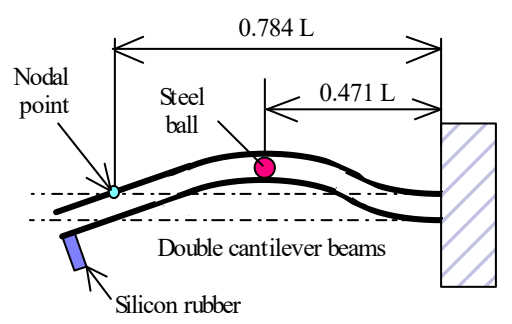

(c) Second vibrational mode.

Figure 1. Structure of proposed motor

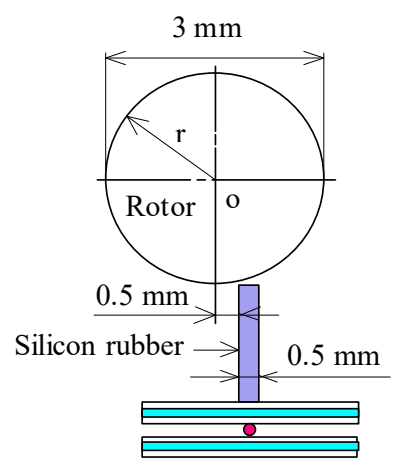

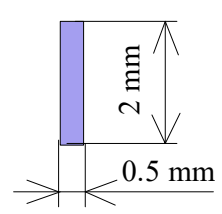

Front view Side view

Detail of silicon rubber

Double bimorph beams

Figure 2. Schematic diagram of silicon rubber and rotor 


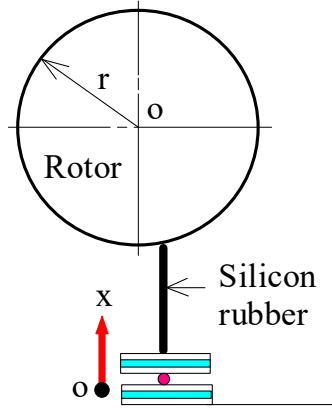

(a) Initial condition

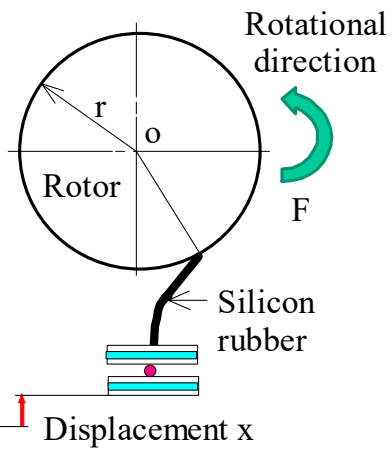

(b) Vibration condition

Figure 3. Rotation due to vibration of two bimorph beams

\section{Basic Output Torque Properties of Motor having two Bimorph Cantilever Beams}

An experiment was conducted using the experimental apparatus shown in Figure 4. In the experiment, two bimorph cantilever beams were vibrated in the resonance condition by inputting a sine wave produced by a function generator into a power amplifier. The resonance frequency for the first vibration mode measured using the experimental apparatus was $338 \mathrm{~Hz}$, and that for the second vibration mode was 2,112 Hz. A commercial motor with diameter of 6 $\mathrm{mm}$ was used to produce a load torque of $19.6 \mu \mathrm{Nm}$ when there was no input current.

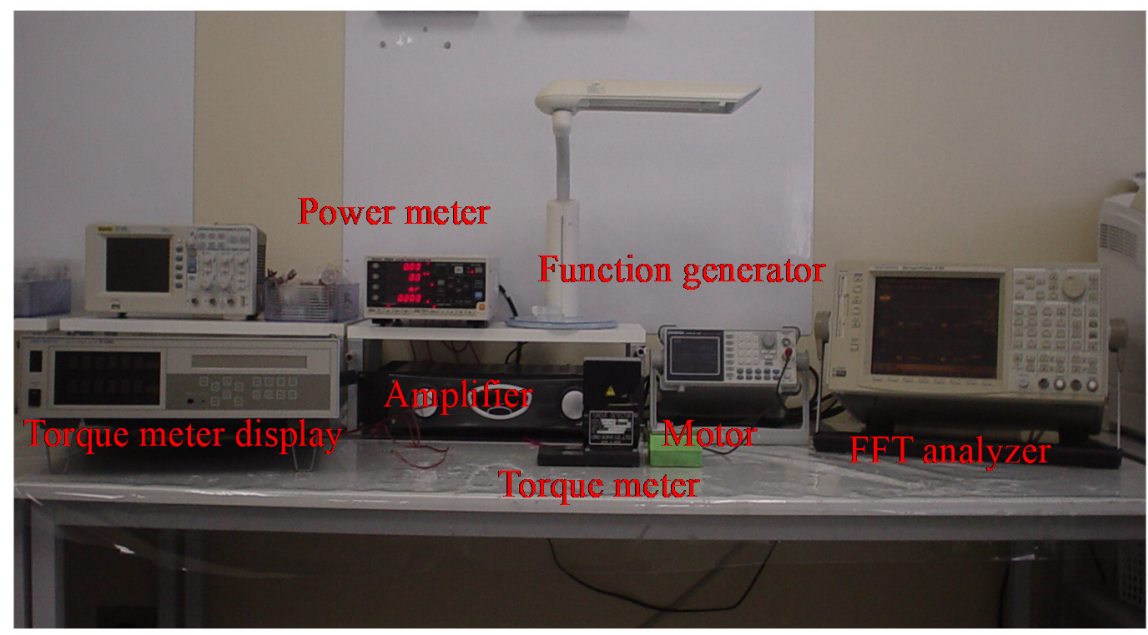

Figure 4. Experimental apparatus

As described above, the two bimorph cantilever beams were connected by a steel ball (diameter: $0.2 \mathrm{~mm}$ ). The connection position for these beams was set to an antinode of the second vibration mode for the cantilever beam. Accordingly, the connection position of the steel ball is $0.471 \mathrm{~L}$, as shown in Figure 1(c). Using the experimental apparatus shown in Figure 4, the rotational properties of the proposed motor were investigated.

Figure 5 shows the relationship between the input voltage to the cantilever beams and the rotational speed. In this case, the rotational speed was linearly proportional to the input voltage. The second vibration mode produces a high output, although the amplitude is small because the resonance frequency of the second vibration mode is high. The maximum rotational speed was $6,804 \mathrm{rpm}$ for an input voltage of $24.6 \mathrm{~V}$.

For the commercial motor, the relationship between the input electric current and the output torque was measured beforehand using a torque meter, with the output torque being changed by varying the input current.

Figure 6 shows the relationship between the output torque and the rotational speed while varying the input power to the cantilever beams. This motor produced an output torque of $39.2 \mu \mathrm{Nm}$ at a rotational speed of 2,182 rpm when the input power was $0.2 \mathrm{~W}$. 


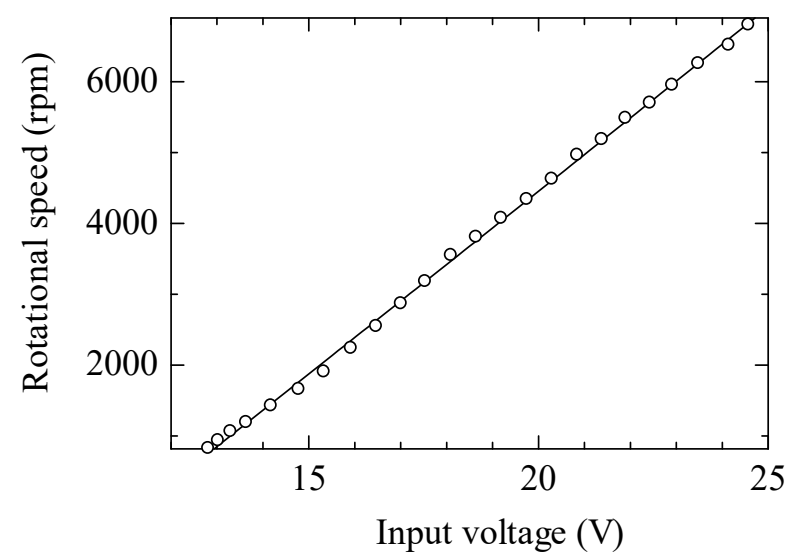

Figure 5. Relationship between input voltage and rotational speed

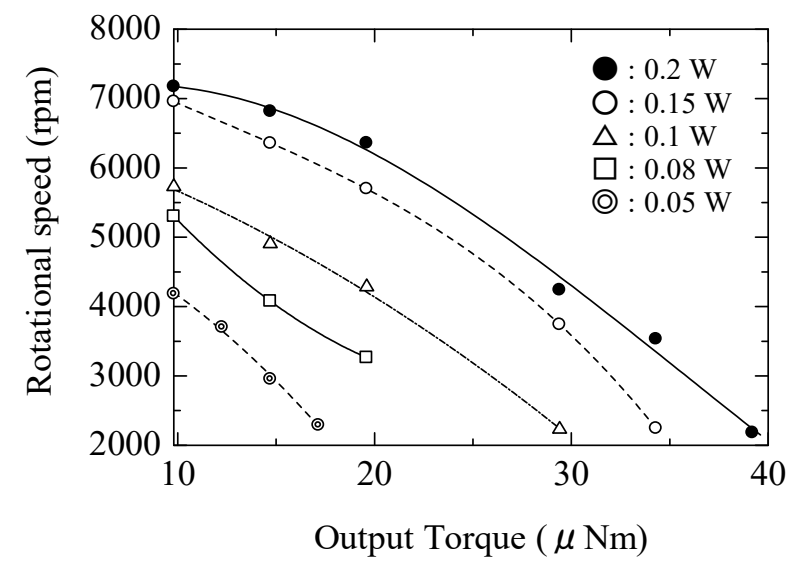

Figure 6. Relationship between output torque and rotational speed

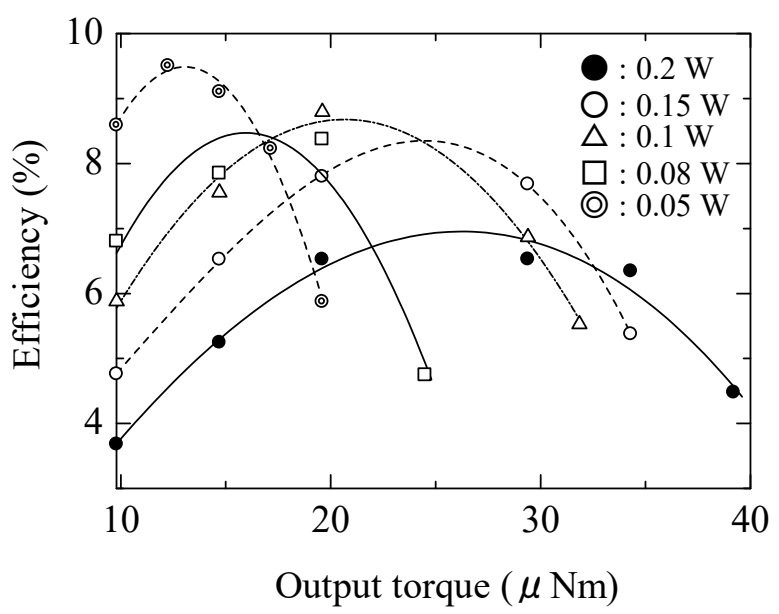

Figure 7. Relationship between output torque and efficiency

Figure 7 shows the relationship between the output torque and the efficiency when the input power into the two bimorph cantilever beams was varied. The maximum efficiency was $9.6 \%$ when the input power was $0.05 \mathrm{~W}$. Based on the results obtained in this experiment, the output torque for this motor not enough. Furthermore, from a practical standpoint, a higher output torque is required. 


\section{Output Torque and Efficiency of Thin-Type Motor having four Bimorph Cantilever Beams}

A new stick-type motor with four bimorph cantilever beams was produced, as shown in Figure 8. Figure 9 shows a photograph of the motor. This motor has two sets of double bimorph cantilever beams. The motor has a length of 20 $\mathrm{mm}$, a height of $6 \mathrm{~mm}$, a width of $5 \mathrm{~mm}$, and a total mass of $2 \mathrm{~g}$. The rotor has a diameter of $3 \mathrm{~mm}$, and a thickness of $3 \mathrm{~mm}$. The diameter of an output axis for this motor is $0.3 \mathrm{~mm}$. In the measurement, the two bimorph cantilever beams were driven by the second resonance frequency of 2,112 Hz. Experimental test was carried out by using the experimental apparatus shown in Figure 4.

Figure 10 shows the relationship between the output torque and the rotational speed while varying the input power to the cantilever beams. The motor produced an output torque of $63.7 \mu \mathrm{Nm}$ when the rotational speed was 1,491 rpm.

Figure 11 shows the relationship between the output torque and the efficiency when the input power to the cantilever beams was varied. The maximum efficiency was $6.2 \%$ when the input power was $0.3 \mathrm{~W}$. For the proposed motor, the volume and weight were reduced by approximately $35 \%$, as compared with a motor from a previous study [16]. Given its small size, this thin-type motor produces a high torque. However, the efficiency is considerably less than that for the motor in the previous study.

Considering the size and weight as the micro-motor by using a thin multilayer cantilever beam, this motor has a very high output. However, the efficiency of this motor is not so high as about $6 \%$. The main reason for the low efficiency is probably the transformation loss of energy due to the silicon rubber through which the resonance energy is transformed into rotational power A high loss is probably not unique to this motor but

inevitable in any motor for which the propulsion relies on highly frictional pressure applied by an elastic material. An optimization of the dimensions and rigidity of the rubber material is required. In addition, it is conceivable to consider increase of torque by electromagnetic force or a hybrid structure of magnetostrictive element and piezoelectric material.

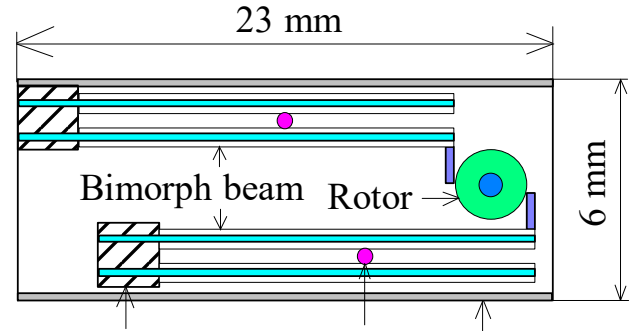

Support stand Steel ball Frame

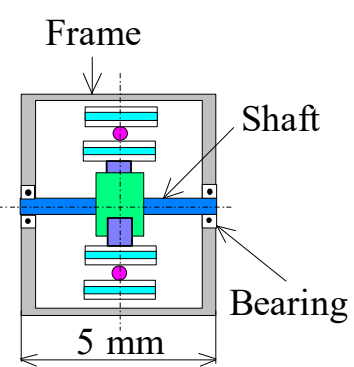

$5 \mathrm{~mm}$

Figure 8. Thin-type motor with four cantilever beams

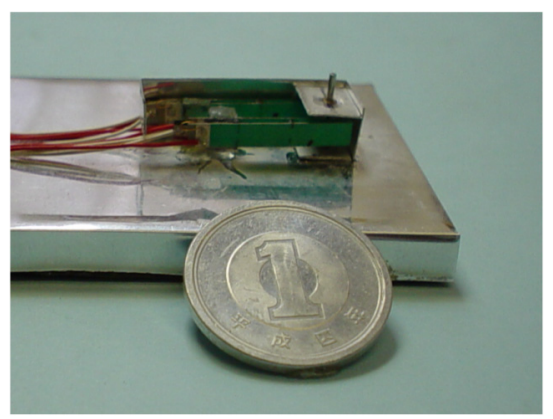

Figure 9. Photograph of motor

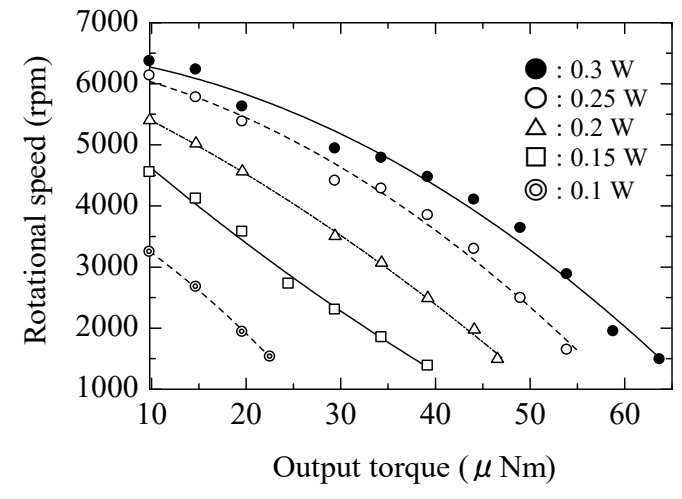

Figure 10. Relationship between output torque and rotational speed

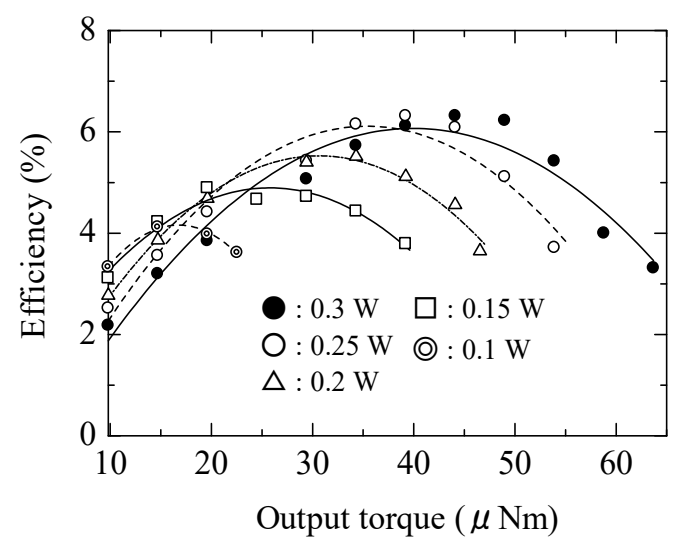

Figure 11. Relationship between output torque and efficiency 


\section{Conclusion}

A non-magnetic thin-type motor based on an electrostatic force and mechanical vibrations was developed using multiple bimorph cantilever beams. The rotational speed of this motor increased in linear to the input voltage when two bimorph beams were used. The greatest value of rotational speed was $6,804 \mathrm{rpm}$ when the input voltage was $24.6 \mathrm{~V}$. Based on these measurement results, a thin-type motor using four bimorph cantilever beams with silicon rubber at their tips was produced. This motor produced an output torque of $63.7 \mu \mathrm{Nm}$ when the rotational speed was $1,491 \mathrm{rpm}$. The maximum efficiency was $6.2 \%$ when the input power was $0.3 \mathrm{~W}$. For its size, the proposed motor produced a significantly high torque. For the proposed motor, the volume and weight were reduced by approximately $35 \%$, as compared with a motor from a previous study.

The entire motor must be miniaturized and made lighter and must incorporate mutual rotation. It is conceivable to consider increase of torque by electromagnetic force or a hybrid structure of magnetostrictive element and piezoelectric material. Future research will be directed toward these goals.

\section{References}

Aoyagi, M., Nakayasu, M., \& Kajiwara, H. (2016). Non-resonance type linear ultrasonic motor using multilayer piezoelastic actuators with parallel beams. Int. J. of Automation Technology, 10(4), 420-424. https://doi.org/doi.org/10(4)=54:2016.7.

Jian, Y., Yao, Z., \& Silberschmidt, V. (2017). Linear ultrasonic motor for absolute gravimeter, ultras, 77. https://doi.org/10.1016/j.ultras.2017.01.023.

Kurosawa, M. (2003). Surface Acoustic Wave Linear Motor. Advanced Robotics, 21, 736-739.

Maruno, T., Honda, T., \& Yamasaki, J. (2000). Considerations on Increase in the Torque of an Electromagnetic Friction-Drive Micro-Motor. Transaction IEE of Japan, 120-A, 289-294.

Morikawa, Y., Ichiki, M., \& Nakada, N. (2003). Electrostatic Optical Motor Using PLZT Elements:Driving Mechanism and Fundamental Experiment. Transactions of the JSME, 69, 2101-2106.

Niino, T., Higuchi, T., \& Egawa, T. (1997). AC Dual Excitation Multiphase Electrostatic Drive. Advanced Robotics, $15,1147-1155$.

Pack, I., Oh, O., Pack, Y., \& Wereley, N. (2013). A Nobel Concept and Proof of Magnetostrictive Motor. IEEE Transaction on Magnetics, 49, 3379-3382. https://doi.org/10.1109/TMAG.2013.2243132.

Peled, G., Yasinov, R., \& Karasikov, N. (2016). Performance and Applications of L1B2 Ultrasonic Motors. Actuators, 5(15). https://doi.org/10.3390/act5020015.

Sharma, S., Nayak, M., \& Dinesh, M. (2008). Shape Memory Alloy Based Motor. Sadhana, 33, 699-712.

Ueno, T. (2009). Miniature spherical motor using iron-gallium alloy (Galfenol). Sensors and Actuators, A154, 92-96.

Xiaolong, L., Junhui, H., Lin, Y., \& Chunsheng, Z. (2014). A novel in-plane mode rotary ultrasonic motor. Chinese Journal of Aeronautics, 27(2), 420-424. https://doi.org/10.1016/j.cja.2013.07.002.

Yaguchi, H. (2015). A New Motor of Stick Type with Coupled Mechanical Vibration of Bimorph Beam and Frictional Force. Engineering, 7, 446 - 454. https://doi.org/10.4236/eng.2015.77040.

Yaguchi, H., Iino, K., \& Eguchi, T. (2014). A New Type of a Magnetic Plane Motor Coupled Mechanical Vibration with Electromagnetic Force. Engineering, 6, 491-499. https://doi.org/10.4236/eng.2014.69051.

Yamamoto, A., Nishijima, T., \& Higuchi, T. (2005). High-Power Double-Sided Drive Type Electrostatic Motor with Stacked-Film Structure. The Japan Society for Precision Engineering, 71, 1245-1249.

Yokota, S., Kuwajima, S., Kondo, Y., \& Edamura, K. (2004). Realization of a Higher Integrated Multi-Layered DPRE Type ECF Micro-Motor. Transactions of Japan Society of Mechanical Engineers, 70, 1463-1469.

Yu, T. (2016). A Novel Ultrasonic Motor Driven by Circumferential Ridge Waves. 2016 International Symposium on Computer, Consumer and Control (IS3C). https://doi.org/doi.ieeecomputersociety.org/10.1109/IS3C.

\section{Copyrights}

Copyright for this article is retained by the author(s), with first publication rights granted to the journal.

This is an open-access article distributed under the terms and conditions of the Creative Commons Attribution license (http://creativecommons.org/licenses/by/4.0/). 